\title{
A Survey on Multisperm Tracking for Sperm Motility Measurement
}

\author{
Priyanto Hidayatullah, Tati L. E. R. Mengko, and Rinaldi Munir
}

\begin{abstract}
Sperm motility is the main criterion in evaluating the quality of semen. Sperm motility measurements can be done in many ways. But the most effective way is to simultaneously track all sperm and calculate the motility parameters of Computer Aided Sperm Analysis (CASA). Based on those parameters, the sperm motility was categorized and the percentage of motile sperm was calculated. This paper presents the analysis of the currently available multisperm tracking methods for sperm motility measurement. In this paper, we discuss why sperm motility is an important parameter for assessing sperm quality and compare several multisperm tracking methods along with an analysis of their advantages and disadvantages. It can be concluded that the main problem in sperm motility measurement is having a good multisperm tracking to obtain precise sperm paths with an efficient computation on semen with high sperm concentrations. If the generated path precision is high, then the CASA parameters calculation results will better describe the actual sperm motility conditions. None of the existing methods can produce precise trajectories in complex cases yet. The complex case is, especially, when sperms collide or cover each other in the situation of large sperm counts appears in one microscope field of view.
\end{abstract}

Index Terms-Multisperm tracking, sperm motility, object tracking, CASA.

\section{INTRODUCTION}

To increase cattle production, artificial insemination (AI) technique is one of the proper alternative solutions. Artificial insemination is a technique of cow breeding by injecting sperm into the cow's womb. This technique is also used in many exporting countries of cattle such as India, France, and Australia. In Australia, for example, about 1.5 million cows are inseminated each year [1]. To support an artificial insemination program, the Indonesian government established Artificial Insemination Centers (Balai Inseminasi Buatan/BIB). This center provides cattle and other good quality livestock semen with a total of 12 centers throughout Indonesia.

Fertilization is largely determined by the quality of semen [2]. Therefore, routine activities undertaken by the AI Center is to evaluate the health of semen before the semen is preserved or inseminated. In addition to evaluating and

Manuscript received August 16, 2017; revised October 10, 2017. This work was supported by Indonesia Endowment Fund for Education (Lembaga Pengelola Dana Pendidikan RI).

Priyanto Hidayatullah, Tati L. E. R. Mengko, and Rinaldi Munir are with School of Electrical Engineering and Informatics, Institut Teknologi Bandung, Jl. Ganesha no. 10 Bandung, Jawa Barat, Indonesia, 40132 (e-mail priyanto@polban.ac.id, tati@stei.itb.ac.id,rinaldi@informatika.org). preserving semen, AI Center also distributes the frozen semen to farming centers.

Semen examination is divided into two groups, namely examination in macroscopic and microscopic. The macroscopic examinations are general semen inspections without the need for complicated tools. The inspections include the volume, semen color, viscosity, and the $\mathrm{pH}$ of the semen. The microscopic examinations are performed to see more detail conditions of the semen where a sophisticated tool needed in the process. These include sperm mass movements, motility, sperm concentration, viability, and sperm morphology [3].

As a case study, in Lembang AI Center, which is the biggest AI Center in Indonesia, the percentage of fresh semen discarded for not passing the macroscopic examinations is at $0.136 \%$ which is very small. While the percentage of fresh semen removed for not passing microscopic examinations is about $30 \%$. As for the frozen semen, $100 \%$ semen passes the microscopic examinations that shows the preservation is well performed. These data suggest that microscopic examinations are more important because in most cases, semen examination is problematic on the microscopic side.

In addition, this data also indicate that the examination of fresh semen requires more attention because semen is more frequent discarded in fresh semen inspections. It is important to note also that the fresh semen concentration is so high that a large number of sperms appear in one field of view. In this situation the occurrence of sperm occlusion and collision is very high. Of the five microscopic examinations, there are two main examinations that are always performed on every examination i.e. motility and sperm mass motion.

The macroscopic examinations can be done easily and quickly manually by a veterinarian or a laboratory technician with good results. Microscopic examination can be done manually, however it takes a long inspection time to get more objective results because we must observe up to 10 fields of view per examination task [3]. In addition, the manual examination has other shortcomings: depending on the experience of the veterinarian [4], the occurrence of human error [4], subjective [5], [6], intra and inter observer variability [7], [8], and causing exhaustion to the observer.

This paper discusses more primarily about the examination of motility. Although sperm mass motion has a close relationship with fertility [5], the related papers are not numerous. As for the measurement of motility, in addition to having a significant role in fertility [4], [9], [10], there are many papers discussing it. Motility measurement is also still a big challenge because the cases listed above have not been handled well.

There are various methods for measuring sperm motility. 
Of the many methods, multisperm tracking simultaneously is the most trusted to deliver results as expected. All motility parameters come from the sperm trajectory formed by the method. If the trajectory can be formed with high precision, then the resulting parameters will have high precision as well. Therefore, the main problem is multisperm tracking to obtain a high precision sperm trajectory [8], [11].

Multisperm tracking sperm has specificity than multiobject tracking in general. Its specificity lies in sperm objects that are very similar to each other both from color, shape, texture, and size. Therefore, a truly reliable tracking method is required to obtain a precise sperm trajectory. In addition to trajectory precision, computational speed is important as motility measurement results are required immediately by the evaluating veterinarian to decide whether the semen being evaluated deserves to be preserved or not.

This paper is arranged by the following arrangement. Section I discusses the background and the objective of the paper. Section II explores in general about CASA. Section III explains more detail about parameters which are used for measuring sperm motility using CASA which called CASA parameters. Section IV analyzes the available multisperm tracking methods along with their summary of advantages and disadvantages. Section V concludes the analysis and give insights for future works.

\section{COMPUTER AIDED SPERM ANALYSIS}

CASA is a system consisting of hardware and software. Since the development of the 1980s, CASA has given promising results [12]. It is estimated that in the future CASA will greatly assist the process of sperm analysis digitally [13]. The expected help keyword from CASA is automatic processing. In addition to being used for human sperm analysis, CASA has been used for the analysis of bull sperm [14] and pig sperm [15].

In general, the CASA system consists of microscope that is given an additional digital camera. This camera will replace the function of the veterinarian eye to record video of the sperm being analyzed. This digital video is then sent to the computer for processing.

The process of digital image processing of sperm is performed in several stages [16]-[18]. First of all, it must be ensured the video taken from the microscope has good quality. To get a good video quality required lighting settings and possibly adding contrast enhancer. In addition, focus settings of the microscope should also be done so that the video is taken with good contrast.

The recording results are stored in the form of a digital video file. In order to be processed, this video must be extracted into image frames. The analysis is done on each frame to identify sperm. After the sperm can be identified, comparison between sequential frames is carried out to obtain sperm motility parameters.

The process of sperm identification is described in [16] with some preprocessing techniques and identification of sperm features. In more detail, the process of sperm image is usually obtained in the form of an RGB image. This image cannot be directly used to recognize sperm. It must, therefore, be converted into binary (black and white) image. The binary image is obtained by applying a threshold on the grayscale version of the RGB image. After having the binary image, edge detection is performed. The edge is required to find the elliptical shape that characterizes the sperm features.

The next stage is sperm tracking. Sperm tracking is performed by mapping between identified sperm on one frame with the identified sperm on the next frame. If the mapping can be well performed, then the trajectory of each sperm can be well drawn which makes it much easier to calculate its CASA motility parameters.

\section{CASA PARAMETERS}

Observation of sperm motion itself is very difficult to do manually because of fast sperm motion. It takes expertise gained from experience long enough to be able to determine the sperm motility. Therefore the existence of CASA is very helpful. In CASA there are parameters that describe sperm motion.

World Health Organization (WHO) defines 9 parameters for measuring the quality of sperm using CASA [19]. These nine parameters are internationally recognized standard parameters. In [9], it was said that sperm concentration and VCL are the most significant parameters in predicting male fertility. While Nagy et al. [10] stated that the Average Path Velocity (VAP) is the most clinically relevant motility parameter of fertility in bull sperm. In short conclusion, there is diversity among researchers considering this issue. For more details, here is a more detailed explanation of the 9 parameters [19]-[21]:

1) Curvilinear velocity (VCL) is the velocity of the sperm along its curve-shaped lane.

2) Straight-line velocity (VSL) is the sperm velocity along the line connecting the starting position and the sperm end position.

3) Average path velocity (VAP), is the average time velocity of the sperm head along the average path. The path is calculated by smoothing the curved trajectory based on the algorithm at CASA.

4) Amplitude of lateral head displacement (ALH) is the farthest distance from the average sperm trajectory

5) Linearity (LIN) is how straight sperm motion.

6) Wobble (WOB) is the actual path oscillation size of the average path, VAP / VCL.

7) Straightness (STR) is linearity to the average path, VSL / VAP.

8) Beat-cross frequency (BCF) is the average value at which the curved path crosses the average path.

9) Mean angular displacement (MAD), is the absolute value of the average time of the rotation angle of the sperm head along the curved path.

\section{Multisperm Tracking Methods}

\section{A. Sperm Trajectory Multidimensional Visualization Methods}

Corkidi et al. [22] built a system to capture the movement 
of sperm in 3 dimensions. This system was a complete system consisting of several components. This system provides video output with dimensions of $512 \times 512$ pixels, frame rate 4200 fps, and a depth of $100 \mu \mathrm{m}$. The semen sample used is sea urchin semen observed in a duration of 1 second.

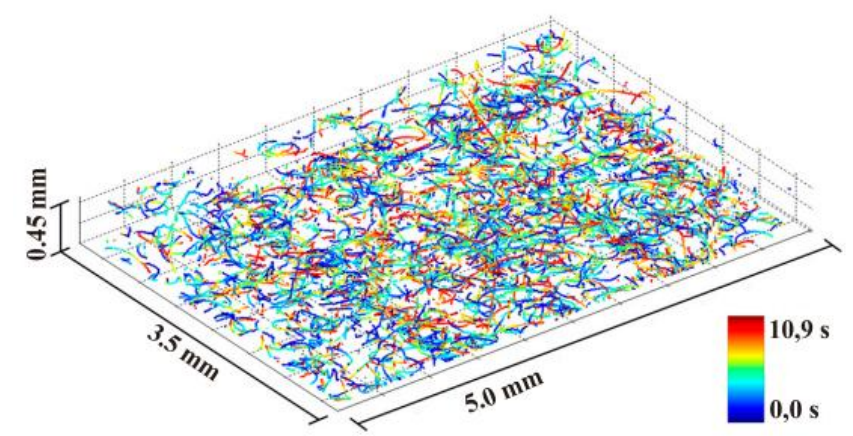

Fig. 1. The reconstructed 3D human sperm trajectories (adapted from [24]).

The trajectory of the sperm contained in the semen can be displayed in 3 dimensions. But there was no explanation about the utilization of this trajectory for measuring the quality of semen. There was no explanation either of the total sperm that exists and how many sperms that are successfully tracked.

Su et al. [23] tackled the problem of sperm dynamic movement visualization on a large volume of semen. They used a lens-free on-chip device which tracked the individual movement of human sperms in 3D. The volume was about 8-17 $\mathrm{mm}^{3}$ with more than 1500 sperms contained. In the previous year, they also published a paper which findings is human sperm's movement formed helical trajectories [24] which illustration is given in Fig. 1. They also quantified some parameters such as VCL, VSL, ALH, BCF, and LIN.

\section{B. Tracking Methods}

Sorensen et al. [25] divided tracking into several stages. The first stage was detection and followed by an estimation of movement and handling of sperm labeling. Sperm detection was carried out using the scale space blob detection method. The basis of using this method was because, in the image of the contrast phase microscope results, $2 / 3$ sperm head looked like a blob (light blob) with an almost circular shape. The position of this bright blob was tracked.

The operator that is commonly used for doing scale space blob detection is Laplacian of Gaussian operator (LoG). At each frame in the video, a scale space blob detector at a certain scale was operated to obtain a filter response on each pixel. This response was converted to the probability of the pixel as the midpoint of the sperm head using the un-normalized Gaussian distribution. Based on the pixel probability map, the sperm position was extracted from the image using a combination of thresholding and connected component analysis.

The process was continued by estimating the sperm movement with two popular methods which are particle filter and Kalman filter. Labeling was done using Hungarian and Hidden Markov Model (HMM) algorithms.

There were three human sperm videos from observation of contrast phase microscope used as samples. The tracking results were measured with Mean Square Error (MSE) and the failure percentage of tracking. The following was the quantitative result of tracking

- Video 1: $\mathrm{MSE}=2.0 ;$ Failed track $=10 \%$,

- Video 2: $\mathrm{MSE}=1.2 ;$ Failed track $=4 \%$,

- Video 3: $\mathrm{MSE}=1.6$; Failed track $=16 \%$.

With this method, the majority of sperm can be tracked properly. However, this method was suitable when the concentration of sperm was not too high. When the sperm concentration was high, the system had difficulty dealing with occlusion. In some cases, objects in the background were considered as sperms. Tracking failure was still considered quite high.

Tomlinson et al. in 2010 [8] used a multitarget algorithm to validate a CASA system. The CASA system features a PC with Windows XP, Fire-I 400 Firewire camera, Firewire capture card, Olympus microscope, and a $37^{\circ} \mathrm{C}$ warm-up table set. Each observation was made on the 20 -mm Leja sliding board. In the algorithm, sperm detection was automatically detected by assigning a threshold to the frame so that the frame converted into a binary image. The objects in the image were then subjected to erosion and dilation operations. Objects whose size was not close to the size of the sperm head were removed. If there were still undesirable artifacts, they were removed manually. The detected sperm objects were then tracked simultaneously using the Markov Chain Monte Carlo (MCMC) method. Based on the trajectory and movement parameters, each sperm was then categorized by its motility based on 1999 WHO laboratory manual [26].

The result was that the system has proximity to manual measurement results, especially for sperm with motility categories a, b, and d. The sperm with motility c was difficult to assess.

In addition to the various advantages, the system ought to still be given manual intervention for the results to be good. The categorization of motility still used the old WHO guidelines [26] so that it needs to be adjusted again with the new WHO guidelines [19].

In 2014, Nurhadiyatna et al. [27] compared several human sperm detection and tracking methods. In fact, this paper more focused to sperm detection comparison. The experiment result itself showed that Gaussian Mixture Model with Hole Filling Algorithm obtains the highest accuracy for human sperm detection compared to other sperm detection methods. For tracking, they used only Kalman filter without comparing to any other tracking method.

Similarly to Nurhadiyatna, Imani et al. [28] in 2014 started the multisperm tracking process with background modeling on the video and subtracted it from the frame so that objects can be extracted. The method used for the background subtraction is 2D-Non-linear Diffusion Filtering. The process continued with morphological operations to get a clearer object. Its multisperm tracking was done by comparing the results of system detection on each frame and calculating its proximity using the Hungarian algorithm.

To measure its accuracy, system results were compared with ground truth data. This method was good for low-density semen (about 10 sperm in one microscope field of view). The 
results showed an accuracy of $96.76 \%$. However, some limitations of this method were the difficulty of handling the following cases:

1. Sperm coming out of the microscope field of view,

2. The missing sperm and reappear in the microscope field of view,

3. The passing sperm,

4. Partial occlusion.

Beya et al. [29] in 2015 performed sperm tracking more gradually. Beginning with a preprocessing stage in which consists of thresholding and contrast stretching. In the preprocessing stage, there was also a potential region detection using the thresholds obtained from entropy maximization and morphological operations with a 10x10 kernel. The region was then represented as a histogram. The histogram was then inputted into Support Vector Machine (SVM).

At the detection stage, all sperm were detected by the bag of words and SVM. While the literature for the bag of words itself used several features, namely: interest points resulted from Speeded Up Robust Features (SURF), Histogram of Oriented Gradients (HOG), and Local Binary Patterns (LBP).

In the tracking phase, potential regions were detected in the first frame. Afterwards, SURF, HOG, and LBP were extracted in the candidate region, which is followed by Mean Shift Tracking.

The system was tested on 3 samples of sheep sperm observation video that already had ground truth data. Experimental results showed the precision of sperm detection of $0.94,0.93$ and 0.96 , and recall of $0.96,0.92$, and 0.97 . Root mean square error (RMSE) in sperm tracking results of 8.06 , 9.01, and 7.09 pixels.

Hidayatullah et al. [17] in 2015 proposed a multisperm tracking for automatic motility measurement. In the first phase, they performed sperm detection using adaptive local threshold and an ellipse detection algorithm which was adjusted according to sperm shape[16]. At the tracking phase, they used Hungarian algorithm based on sperm locations from $k^{\text {th }}$ frame to $k+1^{\text {th }}$ frame. The detection result was encouraging which is useful for tracking phase. Nevertheless, the tracking result was not satisfying enough because position parameter was not enough to predict sperm movement in semen with highly densely populated sperm.

Mahapatra et al. [30] in 2016 proposed a combination method between background subtraction using mixture of Gaussian with a synchronized frame difference. The wavelet was used for reducing noise in advance.

The background subtracted image was then converted into a binary image by making the inter-frame difference. Binary image $B_{1}(x, y)$ was obtained by subtracting between the $\mathrm{k}^{\text {th }}$ and the $k-1^{\text {th }}$ images while binary image $B_{2}(x, y)$ was obtained by subtracting between the $k+1^{\text {th }}$ and the $k^{\text {th }}$ images as described in (1) [30].

$$
\begin{aligned}
& B_{1}(x, y)=\left\{\begin{array}{l}
1, \mid b_{k}(x, y)-b_{k-1}(x, y) \geq T \\
0, \mid b_{k}(x, y)-b_{k-1}(x, y)<T
\end{array}\right\} \\
& B_{1}(x, y)=\left\{\begin{array}{l}
1, \mid b_{k}(x, y)-b_{k-1}(x, y) \geq T \\
0, \mid b_{k}(x, y)-b_{k-1}(x, y)<T
\end{array}\right\}
\end{aligned}
$$

$$
B(x, y)=\left\{\begin{array}{l}
1,\left|B_{1}(x, y) \cap B_{2}(x, y)\right|=1 \\
0,\left|B_{1}(x, y) \cap B_{2}(x, y)\right|=0
\end{array}\right\}
$$

The final frame difference which called three-frame difference $B(x, y)$ was obtained by performing AND operation between $B_{1}(x, y)$ and $B_{2}(x, y)$ as formulated in (2). In the last step, the target was defined if $B(x, y)$ was equal to 1 .

They performed the experiment using microscopic human semen videos with $40 x$ to $400 x$ total magnification. The method itself was implemented using MATLAB 8.0. They claimed that the detection and false alarm rate surpassed the classical mixture of Gaussian model. Unfortunately, this method was not compared to any other state of the art multisperm tracking methods which was already available at the time the paper was written. From the experimental result, false alarm rate was also still considered to be high (21.32\%) and using visual assessment, there were still a lot of undetected sperms.

Jati et al. [31] in 2016 tackled the problem of multisperm tracking on low frame rate video. This was a challenging case as sperm moved very fast and unpredictable. Moreover, the sperm have similar shape and size.

They divided the process into two stages: multisperm detection and multisperm tracking. In the first stage itself, there were three steps which were background subtraction, 2D Gaussian Filter for noise reduction, and thresholding using Otsu's method. The position of the detected sperms was defined by the coordinate of the pixel which has maximal intensity value.

As an additional explanation, the 2D Gaussian Filter was described using (3) [31]

$$
G(x, y)=\frac{1}{2 \pi \sigma^{2}} e^{-\frac{x^{2}+y^{2}}{2 \sigma^{2}}}
$$

where $\sigma$ was defined as the standard deviation of the distribution, while $\mathrm{x}$ and $\mathrm{y}$ were the coordinates of the pixel. The mean distribution was defined as zero. Laplacian of Gaussian $\Delta G(x, y)$ was designed based on Gaussian distribution for blob detection. The last step was performing Laplacian of Gaussian convolution to the input image.

In the case of colliding sperm, standard Kalman Filter performs poorly. To enhance its performance, they combined predicted sperm positions from Kalman Filter and detected positions from multisperm detection stage. Estimated positions were assigned using Hungarian algorithm. The Hungarian algorithm was used with distance (cost) matrices between all sperm pairs which rows were estimated positions and columns were detected positions.

The experiment used two datasets. The first dataset was acquired from Dr. Cipto Mangunkusumo Hospital Lab with frame rate about $20 \mathrm{fps}$ [32] and the second dataset was retrieved from Kokopelli Technology [33]. The result was encouraging with $90 \%$ detection accuracy comparing to visual measurement. For multisperm tracking with three challenging cases: fast motion dataset, low frame rate dataset, and dataset with partial occlusion, they claimed to successfully track the sperms without mentioning the quantification. 
Recently in 2016 Urbano et al. [34] proposed a more reliable and fully automated multisperm tracking method. At the detection stage, the Gaussian filter was used as much as $\mathrm{n}$ times to reduce noise. Afterwards, applying Laplacian of
Gaussian (LoG) / Mexican Hat filter and Otsu thresholding to clarify the existing objects. Subsequently, small objects which size $<5$ pixels were discarded because sperm was unlikely to be that small. The remaining objects were considered sperms.

TABLE I: SUMMARY OF THE ADVANTAGES AND DiSADVANTAGES OF MULTISPERM TRACKING METHODS

\begin{tabular}{|c|c|}
\hline Research & Advantages and disadvantages \\
\hline \multirow[b]{2}{*}{$\begin{array}{l}\text { Corkidi et al., } \\
\text { 2008[22] }\end{array}$} & $\begin{array}{l}\text { Advantages: } \\
\text { - The system was able to visualize trajectories in 3D with only } 1 \text { microscope and } 1 \text { camera } \\
\text { - The track created can be used to analyze sperm motion based on its } 3 \mathrm{D} \text { motion. }\end{array}$ \\
\hline & $\begin{array}{l}\text { Disadvantages: } \\
\text { - It is unclear exactly what benefits can be achieved by visualizing the } 3 \mathrm{D} \text { sperm motion trajectory } \\
\text { - Sperm samples were sea urchins semen that were rarely used in everyday life which bring fewer research benefits. } \\
\text { - There was no explanation either of the total sperm that existed and how many sperms that were successfully tracked. } \\
\text { - Observation duration was only } 1 \text { second. }\end{array}$ \\
\hline \multirow[b]{2}{*}{$\begin{array}{l}\text { Sørensen et al., } \\
2008[25]\end{array}$} & $\begin{array}{l}\text { Advantages: } \\
\text { - Sperm trajectories can be well drawn (average MSE = } 1.6 \text { pixels). }\end{array}$ \\
\hline & $\begin{array}{l}\text { Disadvantages: } \\
\text { - Difficulties occurred in handling occlusion when sperm concentration was high. } \\
\text { - Sometimes the background objects were considered as sperms. } \\
\text { - Tracking failure was still quite high. }(4 \%-16 \%) \text {. }\end{array}$ \\
\hline \multirow[b]{2}{*}{$\begin{array}{l}\text { Tomlinson et } \\
\text { al., 2010[8] }\end{array}$} & $\begin{array}{l}\text { Advantages: } \\
\text { - Experiment settings were set to be as idealistic as possible for reliability. } \\
\text { - The observation was performed by experienced observers. } \\
\text { - The amount of specimens was considerably high }(100) \text {. }\end{array}$ \\
\hline & $\begin{array}{l}\text { Disadvantages: } \\
\text { - Manual intervention was still needed. } \\
\text { - The assumption of hemocytometer results were always correct. } \\
\text { - Manual motility measurement was relatively feasible for sperm categories a, b, and d. Unfortunately, it was difficult for category c. } \\
\text { - Dead sperm that moved due to fluid motion was considered as motile sperm. } \\
\text { - The results of motility calculations by the system were always smaller than manual calculations. }\end{array}$ \\
\hline $\begin{array}{l}\text { Ristic et al., } \\
2011[36]\end{array}$ & $\begin{array}{l}\text { Advantages: } \\
\text { - This measurement method can measure the performance of multisperm tracking methods with results consistent with expectations. } \\
\text { Disadvantages: } \\
\text { - In fact, there was already standard OSPA method previously. This paper added a few enhancements. }\end{array}$ \\
\hline $\begin{array}{l}\text { Su et al. } \\
2012[24] \\
2013[23]\end{array}$ & $\begin{array}{l}\text { Advantages: } \\
\text { - The system can handle numerous sperms }(1500) \text { at once. } \\
\text { - The visualization result was very encouraging. } \\
\text { Disadvantages: } \\
\text { - The visualization capability had not been directly utilized for measuring sperm quality yet. }\end{array}$ \\
\hline $\begin{array}{l}\text { Nurhadiyatna } \\
\text { et al.2014 [27] }\end{array}$ & $\begin{array}{l}\text { Disadvantages: } \\
\text { - Despite its title for comparing both sperm detection and tracking methods, the paper factually more focused on comparing sperm } \\
\text { detection methods. } \\
\text { - The tracking method had not been utilized for measuring sperm quality yet. }\end{array}$ \\
\hline $\begin{array}{l}\text { Imani et al., } \\
2014[28]\end{array}$ & $\begin{array}{l}\text { Advantages: } \\
\text { - The method worked well for semen with low sperm concentration (1-10 sperms per viewing field, 120x, 25fps). } \\
\text { - The research already used the new WHO guidance. } \\
\text { There are limitations on the following cases: } \\
\text { - High sperm concentration semen, } \\
\text { - Sperm that comes out of the field of view, } \\
\text { - Tracking reappear sperms, } \\
\text { - Colliding sperm, } \\
\text { - Partial occlusion. }\end{array}$ \\
\hline \multirow[b]{2}{*}{$\begin{array}{c}\text { Beya et al., } \\
2015[29]\end{array}$} & $\begin{array}{l}\text { Advantages: } \\
\text { - The resulting trajectories have high precision and low RMSE. }\end{array}$ \\
\hline & $\begin{array}{l}\text { Disadvantages: } \\
\text { - Over generalization: The title stated the research used animal samples while factually only sheep samples were used. } \\
\text { - The number of samples were too small ( } 3 \text { videos.) } \\
\text { - The number of sperm in one field of view was small (4-12 sperms). }\end{array}$ \\
\hline \multirow{2}{*}{$\begin{array}{l}\text { Hidayatullah et } \\
\text { al., 2015[17] }\end{array}$} & $\begin{array}{l}\text { Advantages: } \\
\text { - The method has high accuracy of sperm detection which helps tracking phase. } \\
\text { - The tracking method has already been directly used for measuring sperm quality }\end{array}$ \\
\hline & $\begin{array}{l}\text { Disadvantages: } \\
\text { - In the tracking phase, the sperm association accuracy between frames was still relatively low. }\end{array}$ \\
\hline $\begin{array}{l}\text { Arasteh and } \\
\text { Vahdat, } \\
2016[37]\end{array}$ & $\begin{array}{l}\text { Advantages: } \\
\text { - The tools were helpful in the initial test with synthetic datasets } \\
\text { - It has parameters for adding noise, blur. } \\
\text { - The number of sperm generated and its type can be defined by the user. } \\
\text { Disadvantages: }\end{array}$ \\
\hline
\end{tabular}




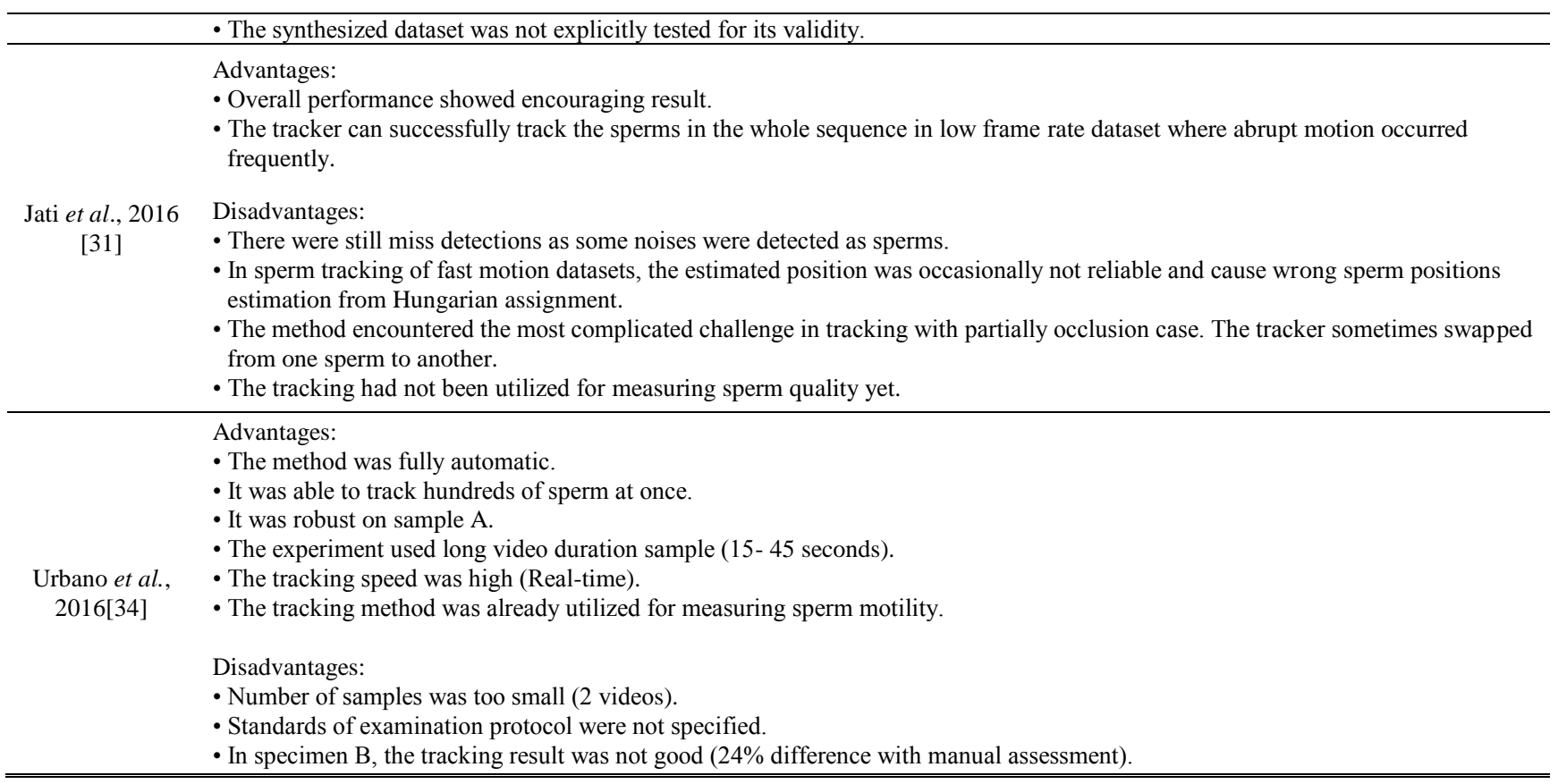

In the tracking phase, the algorithm used was the modified Joint Probabilistic Data Association Filter (JPDAF). After tracking, eight CASA parameters were calculated which were VCL, VSL, VAP, LIN, WOB, STR, ALH, MAD. From that parameters, the sperm motility was measured.

The performance of the method was evaluated using a multiobject tracking algorithm metric called Optimal Sub-Pattern Assignment (OSPA). The experienced technician measures manually and states that there were $92 \%$ motile sperm in sample A and $97 \%$ in sample B. While the developed system showed there were $93 \%$ motile sperm in sample A and $73 \%$ in sample B. In sample A, the system measurement result was very close to the manual measurement result. However, in sample $B$, the measurement result was still too far from the result of manual measurement with $24 \%$ difference.

Experimental results also show that JPDAF and Gaussian Neural Network algorithms consistently outperformed Neural Network and PDAF algorithms. In general, this method has many advantages such as speed (real-time), fully automatic, able to track hundreds of sperm at once and reliable on sample A with a duration of long video samples (15-45 seconds). However, on sample B the accuracy was not encouraging. Fig. 2 shows the reconstructed trajectories.

\section{Performance Measurements of Multisperm Tracking Algorithm Method}

Some researchers focused on the method of measuring the performance of multiobject tracking methods. Of course, this research is very useful. Ristic et al. [36] in 2016 expanded OSPA metrics in measuring the performance of a multiobject tracking algorithm. In this measurement, it was given the mapping between ground truth value with the result of the tracking system and calculated the distance between them using the base distance formula.

Arasteh et al. [37] in 2016 built a system to test two multisperm tracking algorithms. The system was web-based with the purpose of being able to be used by many different platforms. The compared multisperm tracking algorithm was a Kalman filter-based algorithm and a particle filter one. The dataset used in the test was a synthetic dataset.

\section{Summary of Multisperm Tracking Methods}

The currently exist multisperm tracking methods have advantages and disadvantages. Based on the analysis above, here is the summary of the methods mentioned.

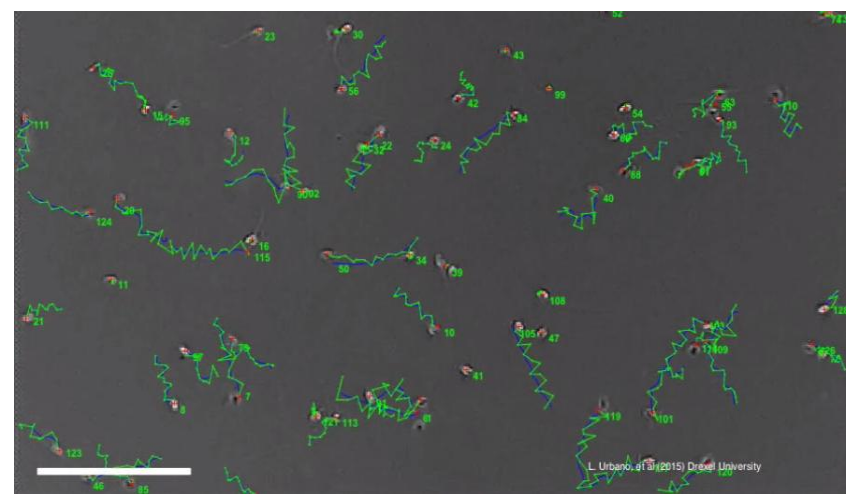

Fig. 2. The reconstructed 3D sperm trajectories [34], [35]

\section{CONCLUSIONS}

Since its introduction in the 1980s, CASA-based semen quality measurements have gained a place because of its urgent presence. But commercial CASA systems are so expensive that stimulate researchers to develop methods that are part of CASA. The major problem that remains unresolved is the multisperm tracking to obtain high precision sperm trajectories with efficient computation on semen with high sperm concentrations. With a high precision trajectory, the CASA parameter calculation results will better describe the actual sperm motility conditions. No existing methods that can actually produce precise trajectories in complex cases, especially colliding sperm and occluding sperm in the situation of large sperm counts appear in one microscope field of view. Even though these cases frequently occur in semen examination at Artificial Insemination Center. Several 
methods of measuring the performance of multisperm tracking algorithms have been developed which include methods developed by Arasteh et al. [37] and Ristic et al. [36].

For future works, this review suggests that multisperm tracking for semen with highly densely populated sperm is very important for sperm motility measurement. Until now, this is still an open problem as none of the current methods could solve it robustly for high number of samples.

\section{REFERENCES}

[1] B. Dunn, G. Fawcett, G. Fahey, D. Boothby, and G. Fordyce, "A practical guide artificial breeding of beef cattle," Queensland Primary Industries and Fisheries, 2009.

[2] J. P. E. Bonde et al., "Relation between semen quality and fertility: a population-based study of 430 first-pregnancy planners," The Lancet, vol. 352, no. 9135, pp. 1172-1177, Oct. 1998.

[3] R. I. Arifiantini, Teknik Koleksi dan Evaluasi Semen pada Hewan. Bogor, Indonesia: IPB Press, 2012.

[4] M. Ravanfar, L. Azinfar, M. H. Moradi, and R. Fazel-Rezai, "Occlusion robust low-contrast sperm tracking using switchable weight particle filtering," Advances in Sexual Medicine, vol. 4, no. 3, pp. 42-54, 2014.

[5] I. David et al., "Mass sperm motility is associated with fertility in sheep," Animal Reproduction Science, vol. 161, pp. 75-81, Oct. 2015.

[6] M. K. Hoogewijs, S. P. De Vliegher, J. L. Govaere, C. De Schauwer, A. De Kruif, and A. Van Soom, "Influence of counting chamber type on CASA outcomes of equine semen analysis: Counting chamber type influences equine semen CASA outcomes," Equine Veterinary Journal, vol. 44, no. 5, pp. 542-549, Sep. 2012.

[7] A. R. Morris, J. R. T. Coutts, and L. Robertson, "A detailed study of the effect of videoframe rates of 25,30 and 60 Hertz on human sperm movement characteristics," Human Reproduction, vol. 11, no. 2, pp. 304-310, 1996.

[8] M. J. Tomlinson et al., "Validation of a novel computer-assisted sperm analysis (CASA) system using multitarget-tracking algorithms," Fertility and Sterility, vol. 93, no. 6, pp. 1911-1920, Apr. 2010.

[9] L. Larsen, "Computer-assisted semen analysis parameters as predictors for fertility of men from the general population," Human Reproduction, vol. 15, no. 7, pp. 1562-1567, Jul. 2000.

[10] Á. Nagy, T. Polichronopoulos, A. Gáspárdy, L. Solti, and S. Cseh, "Correlation between bull fertility and sperm cell velocity parameters generated by computer-assisted semen analysis," Acta Veterinaria Hungarica, vol. 63, no. 3, pp. 370-381, 2015.

[11] A. Arasteh and B. V. Vahdat, "Multiple cell tracking algorithm assessment using simulation of spermatozoa movement," presented at 2015 IEEE 15th International Conference on Bioinformatics and Bioengineering (BIBE), 2015, pp. 1-6.

[12] R. P. Amann and D. Waberski, "Computer-assisted sperm analysis (CASA): Capabilities and potential developments," Theriogenology, vol. 81, no. 1, pp. 5-17.e3, Jan. 2014.

[13] S. T. Mortimer, G. van der Horst, D. Mortimer et al., "The future of computer-aided sperm analysis," Asian Journal of Andrology, vol. 17, no. 4, p. 545, 2015.

[14] P. Kumar, M. Saini, D. Kumar, M. H. Jan, D. S. Swami, and R. K. Sharma, "Quantification of leptin in seminal plasma of buffalo bulls and its correlation with antioxidant status, conventional and computer-assisted sperm analysis (CASA) semen variables," Animal Reproduction Science, vol. 166, pp. 122-127, Mar. 2016.

[15] C. Tretipskul, K. Buranaamnuay, S. Koonjaenak, P. Tummaruk, and M. Techakumphu, "The use of computer-assisted sperm analysis for discriminating series of motility pattern of frozen-thawed boar semen," The Thai Journal of Veterinary Medicine, vol. 40, no. 1, pp. 25-30, 2010.

[16] P. Hidayatullah and M. Zuhdi, "Automatic sperms counting using adaptive local threshold and ellipse detection," in Proc. 2014 International Conference on Information Technology Systems and Innovation (ICITSI), 2014, pp. 56-61.

[17] P. Hidayatullah, I. Awaludin, R. D. Kusumo, and M. Nuriyadi, Automatic Sperm Motility Measurement, pp. 1-5, 2015.

[18] P. Hidayatullah, M. Nuriyadi, I. Awaludin, and R. D. Kusumo, "Pengukuran tingkat motilitas sperma berdasarkan linearitas sperma menggunakan metode adaptive local threshold dan ellipse detection," Jurnal Informatika, vol. 13, no. 2, Feb. 2016.
[19] World Health Organization, WHO Laboratory Manual for the Examination and Processing of Human Semen, 5th ed., Geneva: World Health Organization, 2010.

[20] L. F. Urbano, Robust Automatic Multi-Sperm Tracking in Time-Lapse Images, Drexel University, 2014

[21] L. Witkowski, "Examination of the density of semen and analysis of sperm cell movement," Journal of Medical Informatics \& Technologies, vol. 3, 2002

[22] G. Corkidi, B. Taboada, C. D. Wood, A. Guerrero, and A. Darszon, "Three-dimensional image acquisition system for multi-sperm tracking," in Proc. 5th IEEE International Symposium on Biomedical Imaging, 2008, pp. 145-148.

[23] T.-W. Su, I. Choi, J. Feng, K. Huang, E. McLeod, and A. Ozcan, "Sperm trajectories form chiral ribbons," Scientific Reports, vol. 3, no. 1, Dec. 2013.

[24] T.-W. Su, L. Xue, and A. Ozcan, "High-throughput lensfree 3D tracking of human sperms reveals rare statistics of helical trajectories," Proceedings of the National Academy of Sciences, vol. 109, no. 40, pp 16018-16022, 2012.

[25] L. Sørensen, J. Østergaard, P. Johansen, and M. de Bruijne, Multi-object Tracking of Human Spermatozoa, p. 69142C, 2008.

[26] World Health Organization, WHO Laboratory Manual for the Examination of Human semen and Sperm-Cervical Mucus Interaction, 4th ed. Cambridge, UK New York, NY: Published on behalf of the World Health Organization [by] Cambridge University Press, 1999.

[27] A. Nurhadiyatna, A. L. Latifah, D. Fryantoni, T. Wirahman, R Wijayanti, and F. H. Muttaqien, "Comparison and implementation of motion detection methods for sperm detection and tracking," in Proc. Micro-Nano Mechatronics and Human Science (MHS), 2014 International Symposium on, 2014, pp. 1-5.

[28] Y. Imani, N. Teyfouri, M. R. Ahmadzadeh, and M. Golabbakhsh, "A new method for multiple sperm cells tracking," Journal of Medical Signals and Sensors, vol. 4, no. 1, pp. 35-42, 2014.

[29] O. Beya, M. Hittawe, D. Sidibe, and F. Meriaudeau, "Automatic detection and tracking of animal sperm cells in microscopy images," presented at the Signal-Image Technology \& Internet-Based Systems (SITIS), 2015 11th International Conference on, 2015, pp. 155-159.

[30] S. K. Mahapatra, S. K. Mohapatra, S. Mahapatra, and S. Ghosh, "A gynocology problem solution by tracking multi moving human sperm using wavelet based mixture of Gaussian approach," in Proc. 2016 International Conference on Microelectronics, Computing and Communications (MicroCom), 2016, pp. 1-3.

[31] G. Jati, A. A. Gunawan, S. W. Lestari, W. Jatmiko, and M. H. Hilman, "Multi-sperm tracking using Hungarian Kalman Filter on low frame rate video," in Proc. 2016 International Conference on Advanced Computer Science and Information Systems (ICACSIS), 2016, pp. 530-535.

[32] A. A. S. Gunawan and A. M. Arymurthy, Visual Tracking for Abrupt Motions of Human Sperm Using Smoothing Stochastic Approximate.

[33] Fertilityformen. http://www.fertilityformen.com/infosperm/video.php/

[34] L. Urbano, P. Masson, M. VerMilyea, and M. Kam, "Automatic tracking and motility analysis of human sperm in time-lapse images," IEEE Transactions on Medical Imaging, p. 1, 2016.

[35] Robust automatic multi-sperm tracking in time-lapse images. Vimeo. [Online]. Available: https://vimeo.com/123584621

[36] B. Ristic, B.-N. Vo, D. Clark, and B.-T. Vo, "A metric for performance evaluation of multi-target tracking algorithms," IEEE Transactions on Signal Processing, vol. 59, no. 7, pp. 3452-3457, 2011.

[37] A. Arasteh and B. V. Vahdat, "Evaluation of multi-target human sperm tracking algorithms in synthesized dataset," International Journal of Monitoring and Surveillance Technologies Research, vol. 4, no. 2, pp. 16-29, Apr. 2016

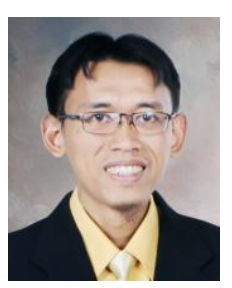

Priyanto Hidayatullah is a doctoral student in School of Electrical Engineering and Informatics, Institut Teknologi Bandung and a lecturer in Computer Engineering and Informatics Department, Bandung State Polytechnic, Indonesia. He received his bachelor degree from the Department of Informatics, Institut Teknologi Bandung, Indonesia in 2004. He received a double degree master of science from Universite Jean Monnet France and the University of Eastern Finland in 2010. He is the book writer titled Pengolahan Citra Digital: Teori dan Aplikasi Nyata. His research interests are biomedical engineering, image processing, computer vision, and machine learning. 


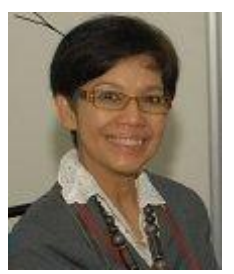

Tati L. E. R. Mengko received the B.Eng. degree in electrical engineering from the Institut Teknologi Bandung (ITB), Bandung, Indonesia, in 1977. In 1985, she obtained the Ph.D. degree from the Institut National Polytechnique de Grenoble at École Nationale Supérieure d'Électronique et de Radioélectricité de Grenoble (ENSERG), France, where she studied about texture-based image processing. Since 2005, she has been a professor with the School of Electrical Engineering and Informatics, ITB. In 2015, she was granted an innovation award from ITB due to her contribution to the development of the device of non-invasive vascular analyzer. She has chaired numerous conferences including International Conference on Instrumentation, Communication, Information Technology, and Biomedical Engineering (ICICI-BME). She is currently the head of Biomedical Engineering Research Group, ITB. Her research interests are biomedical signal and image processing.

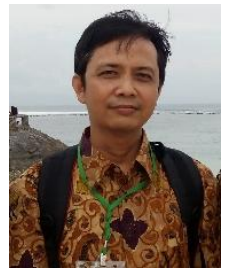

Rinaldi Munir received the B.Eng. degree in informatics engineering from the Institut Teknologi Bandung (ITB), Bandung, Indonesia, in 1992. In 1993, he started his academic career as a lecturer at the Informatics Department, ITB. He obtained M.Sc. degree from ITB in 1999 in digital image compression. In 2010, he obtained the Ph.D. degree from the School of Electrical Engineering and Informatics ITB, where he studied about image watermarking. He is now an associate professor in the School of Electrical Engineering and Informatics, ITB and affiliated with Informatics Research Group. His research interests are cryptography and steganography-related topics, digital image processing, fuzzy logics, and numerical computation. 